\title{
Dynamic Estimation of Visco-Elastic Mechanical Characteristics of Biological Samples under Micro Manipulation
}

\author{
Paolo Di Giamberardino ${ }^{1}$, Maria Laura Aceto ${ }^{1}$, Oliviero Giannini ${ }^{2}$ and Matteo Verotti ${ }^{2}$ \\ ${ }^{1}$ Dept. Computer, Control and Management Engineering Antonio Ruberti, Sapienza University of Rome, Italy \\ ${ }^{2}$ University Niccol Cusano, Via Don Carlo Gnocchi 3, Rome, Italy \\ \{paolo.digiamberardino, marialaura.aceto\}@uniroma1.it, \{oliviero.giannini, matteo.verotti\}@unicusano.it
}

\begin{abstract}
Keywords: Micro Manipulation, Nano Scale Devices, Biological Samples Analysis, Visco-Elastic Characteristic Measurement, Dynamic Parameters Estimation

Abstract: $\quad$ This paper focuses on the possibility of using a recently fabricated micro gripper for the on line estimation of the mechanical characteristics (damping and elasticity) of a sample pinched by the jaws, with particular reference to biological tissues. A classical on line dynamical parameter estimator is computed for the given system with different estimation computations, and its effectiveness has been verified by numerical simulations. Results confirm the feasibility of a micro-robotic clinical device for surgery use equipped with a tissue recognition ability.
\end{abstract}

\section{INTRODUCTION}

Robotics has gained a continuously increasing importance in medical fields, especially in surgery and diagnostic aspects. Great attention has been given to robotic assisted surgery, where the robots in use are of human scale (Fontanelli et al., 2017).

However, there are several cases in which the dimensions of the robotic devices are required to be as small as possible, like in vascular surgery or mini invasive interventions. During these operations, the capability of detecting and identify the tissues under examination, or the different characteristics of part of them, could help the surgeon to perform its task.

One of the most promising technique is based on the estimation of the mechanical characteristics of the samples, specifically their visco-elastic properties.

The possibility of detecting the characteristics of tissues both in surgery and in analysis operations is a strong opportunity for clinical developments. In fact, Literature contains a large number of works confirming the importance of measuring the visco-elastic characteristics of a tissue. Foe example, brain tissues are addressed in (Morrison III et al., 1998), human skin in (Edsberg et al., 2000), reconstituted tissues in (Wakatsuki et al., 2000). In (Guido et al., 2011), the use of dielectrophoretic forces are used to distinguish cell types by means of stretching tests.

The dynamic characteristics of tissues play a fundamental role in the analysis methods, and the results of some tests about the dynamic response of the tissues are reported in (Kiss et al., 2004).

The usefulness of the identification of the tissue elasticity properties during surgical operations is discussed in (Tavakoli et al., 2006). For example, the relationships between elastic and viscoelastic properties of undifferentiated adipose-derived stem cells and lineage-specific metabolite production are studied in (González-Cruz et al., 2012). To perform these tasks, additional sensors like vision systems can also be implemented (Boonvisut and Çavuşoğlu, 2013).

In order to better investigate the characterization and the influence of the mechanical response of tissues in physiological or pathological aspects, the development of mathematical models plays an important role for successful results. A review of this aspect can be found in (Choi, 2016), whereas mechanical concepts can be applied to interpret deformation profile of aspirated soft tissues, as in (Nava et al., 2004; Nava et al., 2008).

All the recalled examples show the importance of being able to recognise the mechanical characteristics of a tissue, often at a micro or nano scale. Displacement and force sensors can help for acquiring measurements to be used for system identification.

Parameter estimation problems are not limited to small scale systems; the often arise in robotic control applications. Still referring to medical systems, for example in (Wilkening and O., 2014), the estimation of mass parameters for a safe and comfortable 
human-robot interaction is considered for robot assisted rehabilitation of patients after surgical interventions.

In (Cao et al., 2015), a two arms nursing care robot is studied. The knowledge of mechanical parameter as the contact points between the two arms and a carried body, or the full system center of gravity during body manipulation, plays an important role for a efficient and effective control operations. Then, the possibility of estimating the center of mass position in different cases of contact situation is studied.

These examples, among many others, in several fields of robotic applications are a valid demonstration of the importance and the interest in the parameter estimation problems.

Several solutions are available for parameter estimation of dynamical systems, depending on the characteristics of the sensor signals, the linearity or the non linearity of some relationships, and other structural properties of the model.

In this work, the possibility of using a microgripper for the evaluation of the mechanical characteristics of biological samples is presented, considering an estimation algorithm for the viscosity and elastic parameters under generic control torques. The paper is organised as follows. The microdevice is introduced in Section 2, whereas the mathematical model is discussed in Section 3. The adopted identification algorithms are described in Section 4, and some numerical results are reported and discussed in Section 5.

\section{THE EXPERIMENTAL DEVICE}

The microsystem considered in this paper, depicted in Figure 1, is described in detail in (Bagolini et al., 2017) and (Di Giamberardino et al., 2018). The device is fabricated as a silicon monolithic structure, arranged with a comb drive at the anchor of each jaw. The comb actuators exert the input torques that, through the deflections of a flexure hinge (Verotti et al., 2015; Verotti et al., 2017), move the jaws during the gripping tasks (Cecchi et al., 2015). As reported in (Bagolini et al., 2017), the fabrication method adopted makes use of Deep Reactive-Ion Etching applied on Silicon on Insulator wafer.

The operative situation is drawn in Figure 1, in which the sample is pinched by the gripper and is kept between the jaws. The points $A$ and $D$ represent the hinges/actuators, while the points $B$ and $C$ are the contact points between the jaws and the sample.

The operational problem has been already investigated in (Bagolini et al., 2018) and (Di Giamberardino et al., 2018), where an estimation of the elas-

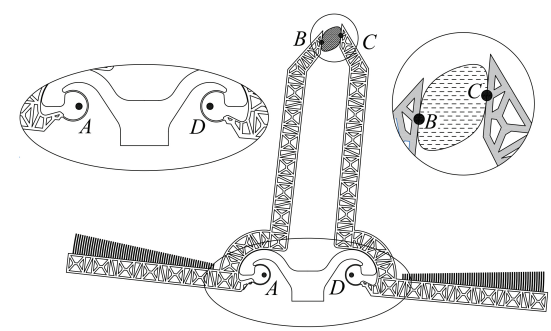

Figure 1: The gripping system in a generic configuration.

tic and viscous coefficients of the mechanical model of the sample was provided during simulations with input signals of suitable waveform. In particular, in (Bagolini et al., 2018), the elastic coefficient was obtained gripping the sample by actuating the comb drive connected to the first jaw, until the second comb drive reached a predefined angular displacement (sufficiently small to guarantee the safeness of the sample). This particular actuation choice arises from the fact that the device is not equipped with a force or torque sensor. The hypothesis of gripper joints characterized by a lower elastic coefficient than the one of the sample should assure that, for small joint angle displacements, the gripping force would not be dangerously high.

This critical action is provided by means of a feedback control scheme in which the safe displacement of the second joint is the reference signal. The elastic coefficient is then computed, at steady state conditions, from the measurement of the jaws angular displacements.

The measurement scheme was improved in (Di Giamberardino et al., 2018), in order to estimate also the viscosity coefficient. At the basis of the computation there is the almost linearity conditions under small and slow state variables evolution. Then, a sinusoidal input of sufficiently small amplitude was added to the first joint. The viscous coefficient was obtained by making use of the linearised dynamics or of the nonlinear characteristics of the viscosity as a function of the frequency of the input torque.

In this paper, the possibility of using an estimation algorithm for the viscosity and elastic parameters of the crimped sample under generic control torques is presented.

The algorithm is based on the particular structure of the mathematical model: despite its general non linearity, it results linear with respect to the unknown parameters. Section 3 is devoted to a brief recall of the mathematical model which is used in Section 4 to design the estimator system. 


\section{THE MATHEMATICAL MODEL}

The mathematical model refers to the case of full contact condition between the jaws and the sample at points $B$ and $C$ of Figure 1, i.e. during the measurement phase. Then, the quadrilateral $A B C D$ constitute a closed chain composed by the links $A D$ (the base), $A B$ and $C D$ (the jaws), and $B C$ (the sample). All the links have a fixed length with the exception of the sample dimension $B C$, whose compression is at the basis of the measurement. The different materials constituting the device and the sample is at the basis of the assumption that the stiffness of the jaws is much greater than the one of the sample.

For sake of simplicity in the model representation, all the considered variables are referred to an initial condition in which the gripper, in a symmetrical configuration, is in touch without deformation of the sample.

Then, following the notation introduced in (Di Giamberardino et al., 2018) and with reference to Figure 1 , the orientation of the two jaws are denoted by $\tilde{\theta}_{i}$, and the reference values by $\hat{\theta}_{i}$. Therefore, the angles $\theta_{i}=\tilde{\theta}_{i}-\hat{\theta}_{i}$ represent the relative angular displacements of the two links from their neutral configuration, with $i=2$ for the left link and $i=4$ for the right one.

Simple geometric considerations give $\hat{\theta}_{2}=\pi-\hat{\theta}_{4}$.

Considering the two contact points $B$ and $C$, the orientation of the line $B C$ has the same notation, with $i=3$ and $\hat{\theta}_{3}=0$. The angles are defined according to the counter clockwise rule. The reference value for the distance $B C$, corresponding to zero elastic reaction force of the sample, is denoted by $\hat{u}$, and its actual value by $\tilde{u}$. Therefore, the deformation is equal to $u=\tilde{u}-\hat{u}$.

The values of the variables in the neutral condition are given in Table 1.

Table 1: Constants.

\begin{tabular}{|c|c|}
\hline Parameter & Numerical value \\
\hline$\hat{\theta}_{2}$ & $1.44 \mathrm{rad}$ \\
$\hat{\theta}_{4}$ & $1.70 \mathrm{rad}$ \\
$\hat{u}$ & $150 \cdot 10^{-6} \mathrm{~m}$ \\
\hline
\end{tabular}

The following parameters are defined:

i. $l$ is the common length of the two links which constitutes the jaws, i.e. the distances $A B$ and $C D$;

ii. $d$ is the distance between the hinges $(A D)$;

iii. $k_{2}, k_{4}$ and $K$ are the torsional stiffness of the two jaws and the stiffness coefficient of the tissue sample, respectively; iv. $c_{2}, c_{4}$ and $c$ are the viscous damping coefficients of the two jaws and of the sample, respectively;

v. $I_{2}$ and $I_{4}$ are the two jaws moments of inertia around $A$ and $D$;

vi. $\tau_{2}$ and $\tau_{4}$ are the input torques generated by the comb drives.

The symmetry of the neutral configuration implies the relationship $\hat{u}=d-2 l \cos \hat{\theta}_{2}$.

The angular dynamical model of each of the two links can be computed. For the first joint, with subscript 2, from the torque balance condition and assuming the inertia of the sample negligible, one has

$$
\begin{aligned}
I_{2} \ddot{\theta}_{2}= & -c_{2} \dot{\theta}_{2}-k_{2} \theta_{2}-c l \sin \left(\tilde{\theta}_{2}-\theta_{3}\right) \dot{u} \\
& -k l \sin \left(\tilde{\theta}_{2}-\theta_{3}\right) u+\tau_{2},
\end{aligned}
$$

while for the second one, with subscript 4 , the corresponding expression is

$$
\begin{aligned}
I_{4} \ddot{\theta}_{4}= & -c_{4} \dot{\theta}_{4}-k_{4} \theta_{4}+c l \sin \left(\tilde{\theta}_{4}-\theta_{3}\right) \dot{u} \\
& +k l \sin \left(\tilde{\theta}_{4}-\theta_{3}\right) u+\tau_{4} .
\end{aligned}
$$

For the device here considered, the values of the parameters appearing in (1) and (2) are reported in Table 2. These values, together with the ones in Table 1 , can help to figure out the whole dimension of the gripper.

Table 2: Numerical values of the parameters.

\begin{tabular}{|c|l|}
\hline Parameter & Numerical value \\
\hline$d$ & $5.47 \cdot 10^{-4} \mathrm{~m}$ \\
$l$ & $1.496 \cdot 10^{-3} \mathrm{~m}$ \\
$I_{2}, I_{4}$ & $1.25 \cdot 10^{-14} \mathrm{~kg} \mathrm{~m}^{2}$ \\
$k_{2}, k_{4}$ & $0.30 \cdot 10^{-6}\left(\mathrm{Kg} \mathrm{m}^{2}\right) /\left(\mathrm{s}^{2} \mathrm{rad}\right)$ \\
$c_{2}, c_{4}$ & $1.24 \cdot 10^{-12}\left(\mathrm{Kg} \mathrm{m}^{2}\right) /(\mathrm{s} \mathrm{rad})$ \\
\hline
\end{tabular}

The system has two degrees of freedom and then it is fully described by equations (1) and (2). In fact, since the closed kinematic chain configuration is considered, it is possible to compute the remaining variables $\theta_{3}$ and $u$ and their time derivatives as functions of the state variables $\theta_{2}, \theta_{4}, \dot{\theta}_{2}$ and $\dot{\theta}_{4}$.

Such computations are reported in (Di Giamberardino et al., 2018) and follow geometric considerations on the quadrilateral $A B C D$. The results are here reported:

$$
\begin{gathered}
\theta_{3}=\arctan \frac{-l \sin \tilde{\theta}_{2}+l \sin \tilde{\theta}_{4}}{d-l \cos \tilde{\theta}_{2}+l \cos \tilde{\theta}_{4}} \\
\tilde{u}=\sqrt{\left(d-l \cos \tilde{\theta}_{2}+l \cos \tilde{\theta}_{4}\right)^{2}+\left(l \sin \tilde{\theta}_{4}-l \sin \tilde{\theta}_{2}\right)^{2}} \\
u=\tilde{u}-\hat{u} \\
\dot{u}=\dot{\theta}_{2} l \sin \left(\tilde{\theta}_{2}-\theta_{3}\right)-\dot{\theta}_{4} l \sin \left(\tilde{\theta}_{4}-\theta_{3}\right)
\end{gathered}
$$

An interesting observation is that the computation of $\dot{\theta}_{3}$ is not required. 


\section{MECHANICAL \\ CHARACTERISTICS ESTIMATION OF SAMPLES}

As discussed in the Introduction, important aspects when dealing with robotics in surgery are the recognition of the different tissues, as well as the different characteristics of parts of the same tissue. Robotic assisted tissue identification and characterization for a pure diagnostic purpose is another important aspect that is usually addressed.

As recalled in Section 2, a measurement scheme has been proposed for the elastic properties (Bagolini et al., 2018) and then for the viscous characterization (Di Giamberardino et al., 2018). The necessity of particular input signal waveforms to perform such measurements is compatible with clinical diagnostic tests applications, but it is not suitable for a tissue manipulations during surgery operations. This latter case implies the adoption of a measurement scheme able to work under any input and operative conditions. Therefore, an on line dynamical estimator is proposed as a more efficient strategy for the sample characterization.

A simple approach to system parameters identification makes use of numerical solutions based on recursive least square methods. The condition under which this kind of techniques can be successfully applied is that the set of parameters to be estimated appear linearly in the dynamics. In fact, in this case, the model can be rearranged in the form of a linear time varying systems in which the parameters to be identified are the unknowns, and all the other terms are function of the state and of the output variables, supposed measurable. This technique has been fruitfully used in several applications, as for example in ((Flacco et al., 2011; Lundquist and Schön, 2009; Vahidi et al., 2005; Lee and Jung, 2016)).

The structure in which the dynamics has to be rewritten is

$$
y_{i}(t)=M_{i}(t) \omega_{i}(t), \quad i \in[1, \ldots, m],
$$

where $m$ are the degrees of freedom of the system. In our case, $m=2$, making reference to equations (1) or (2). The term $\omega_{i}(t)$ is one of the of unknown parameters, whereas $y_{i}(t)$ and $M_{i}(t)$ are known quantities coming from the dynamics expressions.

All the quantities in (6) are time varying, since they are computed during the dynamics evolution; this means that also $\omega_{i}(t)$, despite it is referred as the parameters vector, is a function of time because the estimated values change at each update of the procedure, converging to the constant values of the parameters.
The application of this approach needs the dynamical equations (1) and (2) be rewritten in a linear form with respect to the unknown parameters. According to the notation in (6), one can set

$$
\begin{aligned}
& y(t)=\left(\begin{array}{l}
y_{1}(t) \\
y_{2}(t)
\end{array}\right)=\left(\begin{array}{l}
I_{2} \ddot{\theta}_{2}+c_{2} \dot{\theta}_{2}+k_{2} \theta_{2}-\tau_{2} \\
I_{4} \ddot{\theta}_{4}+c_{4} \dot{\theta}_{4}+k_{4} \theta_{4}-\tau_{4}
\end{array}\right)(7) \\
& M(t)=\left(\begin{array}{l}
M_{1}(t) \\
M_{2}(t)
\end{array}\right)=\left(\begin{array}{c}
-l \sin \left(\tilde{\theta}_{2}-\theta_{3}\right) \\
l \sin \left(\tilde{\theta}_{4}-\theta_{3}\right)
\end{array}\right)\left(\begin{array}{ll}
\dot{u} & u
\end{array}\right)(8) \\
& \omega(t)=\left(\begin{array}{l}
\omega_{1}(t) \\
\omega_{2}(t)
\end{array}\right)=\left(\begin{array}{l}
c(t) \\
k(t)
\end{array}\right)
\end{aligned}
$$

A recursive least squares (RLS) filtering algorithm is adopted for the on-line identification of the viscous damping and the stiffness coefficient of the tissue sample.

Referring to (7)-(9), the general expressions to be defined for a generic recursive least squares (RLS) filtering algorithm are (Ljung, 1999):

$$
\begin{aligned}
\hat{\omega}_{i}(t) & =\hat{\omega}_{i}(t-1)+K_{i}(t) \varepsilon_{i}(t) \\
\varepsilon_{i}(t) & =y_{i}(t)-\hat{y}_{i}(t) \\
\hat{y}_{i}(t) & =\phi_{i}^{T}(t) \hat{\omega}_{i}(t-1) \\
K_{i}(t) & =Q_{i}(t) \phi_{i}(t)
\end{aligned}
$$

for $i=1,2$, where $\hat{\omega}_{i}(t)$ and $\hat{y}_{i}(t)$ are the current estimation values of $\omega_{i}(t)$ and $y_{i}(t), \varepsilon_{i}(t)$ is the current prediction error, the gain $K_{i}(t)$ determines how much the prediction error affects the update in the parameters estimation, and $\phi_{i}(t)$ represents the gradient of the predicted model output with respect to $\omega_{i}(t)$.

The RLS filtering algorithm is applied considering different ranges of values of the parameters to be estimated, in order to show how much the different viscous and elastic characteristics of the dynamical system affects the convergence of the algorithm and the steady state behaviour.

\subsection{Forgetting factor based RLS estimator}

The estimation method adopted is a forgetting factor based RLS algorithm. In equations (10), the following choices are performed

$$
Q_{i}(t)=\frac{P_{i}(t-1)}{\lambda+\phi_{i}^{T}(t) P_{i}(t-1) \phi_{i}(t)},
$$

where

$$
P_{i}(t)=\frac{1}{\lambda}\left(P_{i}(t-1)-R_{i}(t)\right)
$$

and

$$
R_{i}(t)=\frac{P_{i}(t-1) \phi_{i}(t) \phi_{i}^{T}(t) P_{i}(t-1)}{\lambda+\phi_{i}^{T}(t) P_{i}(t-1) \phi_{i}(t)} .
$$


$i=1,2$. It is assumed that the residual $\varepsilon_{i}(t)$ (the difference between the estimated and the measured value for $\left.y_{i}(t)\right)$ is affected by a white noise with covariance equal to 1.

According to previous equations, the $\hat{\omega}_{i}(t)$ are computed in order to minimize the sum of residuals squares

$$
\hat{\omega}_{i}(t)=\underset{\theta}{\arg \min } \sum_{k=1}^{t} \lambda^{t-k} \varepsilon_{i}^{2}(t)
$$

In (11), (12), (13) and (14), $\lambda \in R$, is the so-called forgetting factor. It is introduced in order to consider differently the time sequence of the errors $\varepsilon_{i}(t)$, according to an exponentially decreasing weight if $\lambda \in(0,1)$. This choice is effective in case of time varying parameters. When dealing with constant parameters, the choice $\lambda=1$ is usually adopted.

The algorithm (10), with positions (11)-(13), has been applied to the case here considered. In all the simulations, the initial values of the parameters have been chosen quite differently from the real values. The initial covariance, proportional to $P_{2}$, has been fixed taking into account that the covariance matrix has to be chosen according to a priori knowledge of the parameters at $t=0$ : very high values of the covariance matrix elements correspond to almost completely unknown parameters.

Remark: note that the forgetting factor method is a particular case of the Kalman filter.

\section{SIMULATIONS}

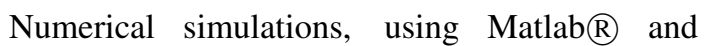

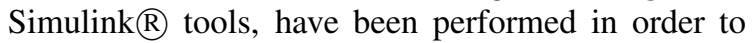
show effectiveness, benefits and differences of the proposed estimation methods.

Three numerical cases are considered. The first one corresponds to a realistic case with elastic and damping coefficient much greater than the ones of the mechanical structure, with $c=8.4 \cdot 10^{-6} \mathrm{~N} \mathrm{~m} \mathrm{~s} / \mathrm{rad}$ and $k=2.5 \cdot 10^{-3} \mathrm{~N} \mathrm{~m} / \mathrm{rad}$, and with the elastic coefficient greater than the damping one.

The second one has been chosen considering a sample with a damping coefficient greater than the elastic one in order to check, by comparison, the dependency of the algorithm convergence from the two different mechanical characteristics. The order of magnitude for the two coefficients have been exchanged, getting $c=8.4 \cdot 10^{-3} \mathrm{~N} \mathrm{~m} \mathrm{~s} / \mathrm{rad}$ and $k=$ $2.5 \cdot 10^{-6} \mathrm{~N} \mathrm{~m} / \mathrm{rad}$.

The last choice has been performed in order to check the algorithm behaviour for a very poorly damped sample. In fact, the dumping coefficient is assumed as $c=8.4 \cdot 10^{-11} \mathrm{~N} \mathrm{~m} \mathrm{~s} / \mathrm{rad}$ while $k=$ $2.5 \cdot 10^{-5} \mathrm{~N} \mathrm{~m} / \mathrm{rad}$.

The initial parameters values, for all the simulations, have been chosen as $c(0)=10^{-9} \mathrm{~N} \mathrm{~m} \mathrm{~s} / \mathrm{rad}$ and $k(0)=10^{-7} \mathrm{~N} \mathrm{~m} \mathrm{/rad}$.

For the forgetting factor RLS estimator, the $2 \times 2$ square covariance matrix has been set as a diagonal matrix, with both the diagonal elements equal to $10^{20}$, while the forgetting factor $\lambda$ is fixed to $\lambda=0.99$.

Simulation results obtained for the first case $(c=$ $8.4 \cdot 10^{-6} \mathrm{~N} \mathrm{~m} \mathrm{~s} / \mathrm{rad}$ and $k=2.5 \cdot 10^{-3} \mathrm{~N} \mathrm{~m} / \mathrm{rad}$ ) are depicted in Figure 2 for the damping coefficient $c$, and in Figure 3 for the elastic one $k$.

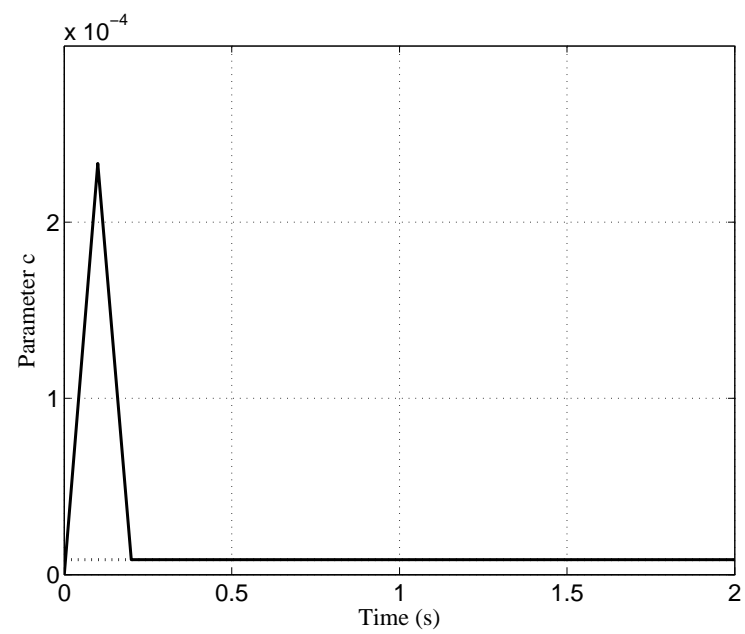

Figure 2: Time evolution of the estimated value $c$.

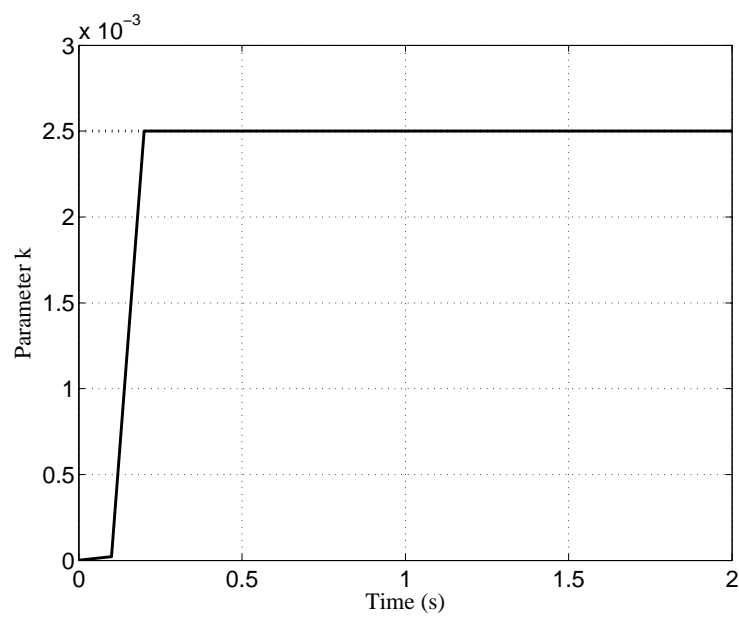

Figure 3: Time evolution of the estimated value $k$.

The solid lines denote the estimations evolution, the dotted lines correspond to the true values of the parameters, plotted as a reference. As expected, the algorithm converges. Moreover, from Figures 4 and 5 that depict the estimation errors for parameter $c$ and 


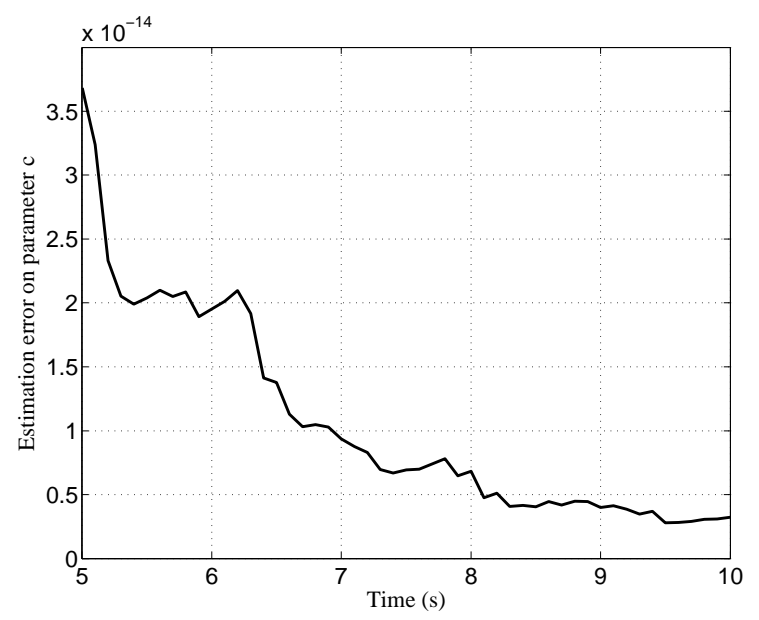

Figure 4: Estimation error on parameter $c$ after the transient.

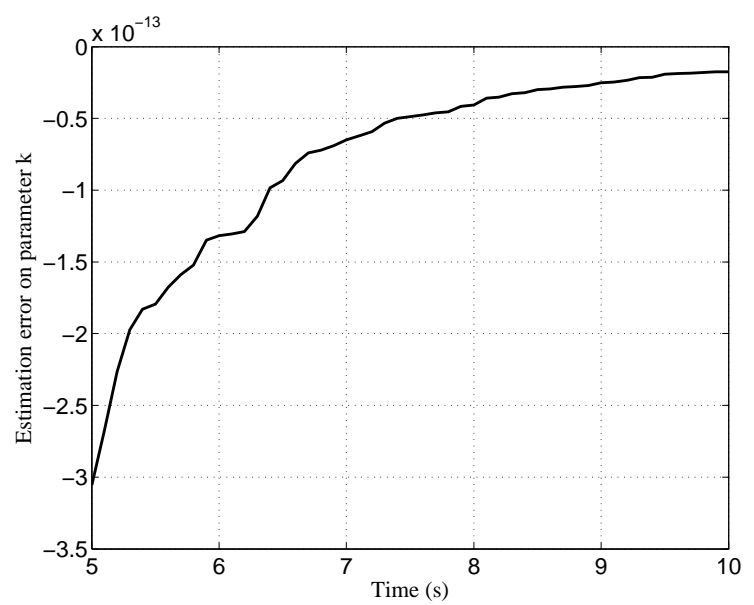

Figure 5: Estimation error on parameter $k$ after the transient.

$K$ respectively after $5 \mathrm{~s}(t \in[5,10] s)$, it is possible to observe that the convergence is very fast, being the order of magnitude of the errors $10^{-14}$ for $c$ and $10^{-13}$ for $k$.

For the second case $\left(c=8.4 \cdot 10^{-3} \mathrm{~N} \mathrm{~m} \mathrm{~s} / \mathrm{rad}\right.$ and $k=2.5 \cdot 10^{-6} \mathrm{~N} \mathrm{~m} / \mathrm{rad}$ ), the simulation results are depicted in Figure 6 for the damping coefficient $c$, and in Figure 7 for the elastic one $k$. Again, the solid lines refer to the estimations evolution while the dotted ones are the true reference values.

Also in this case the fast convergence of the algorithm can be confirmed observing Figure 8 and Figure 9: the order of magnitude of the errors, after $5 \mathrm{~s}$, $(t \in[5,10] s)$, is equal to the previous case.

The results obtained by simulation of the third case $\left(c=8.4 \cdot 10^{-11} \mathrm{~N} \mathrm{~m} \mathrm{~s} / \mathrm{rad}\right.$ and $k=2.5 \cdot 10^{-5} \mathrm{~N}$ $\mathrm{m} / \mathrm{rad}$ ) are reported in Figure 10 for the damping coefficient $c$, and in Figure 11 for the elastic one $k$, where the solid lines mark the estimations evolution and the dotted lines denote the true values of the parameters.

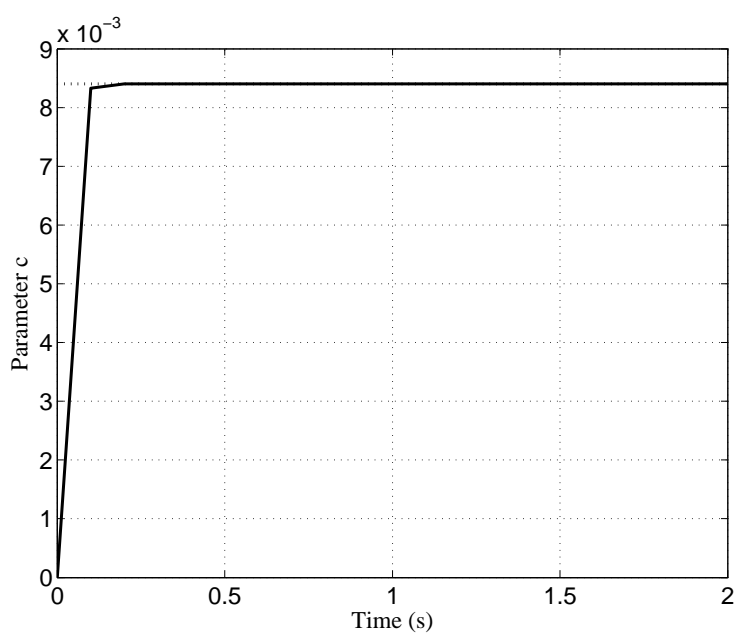

Figure 6: Time evolution of the estimated value $c$.

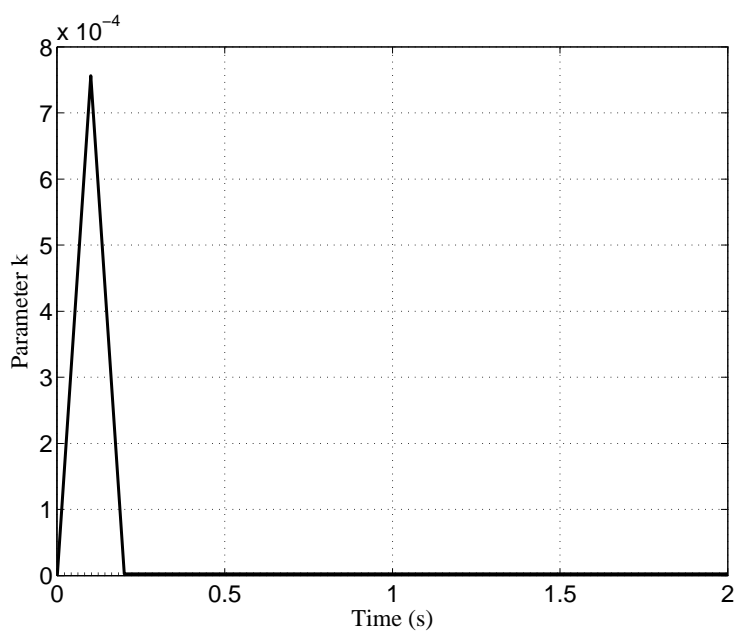

Figure 7: Time evolution of the estimated value $k$.

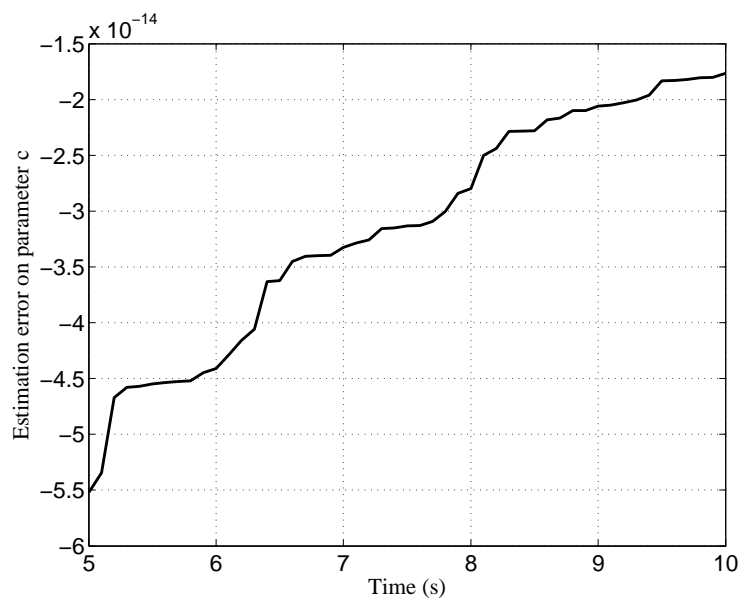

Figure 8: Estimation error on parameter $c$ after the transient.

The time evolutions of the errors are plotted in 


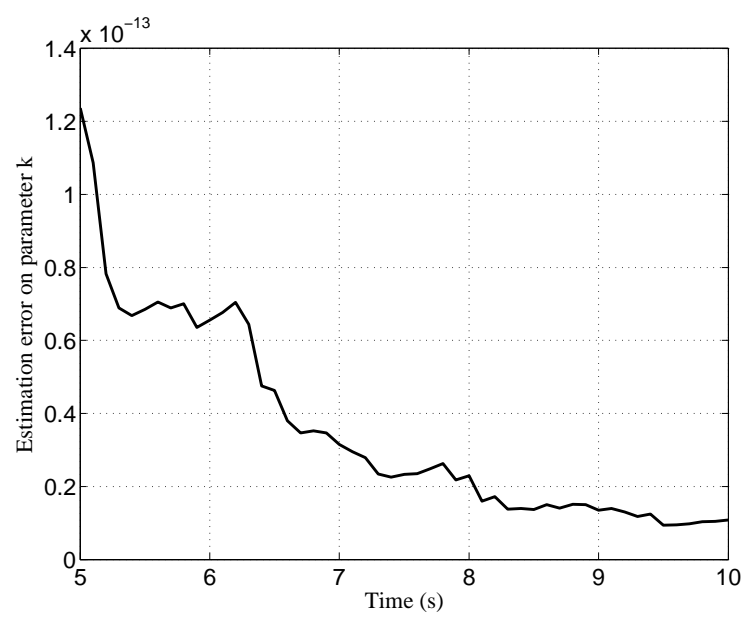

Figure 9: Estimation error on parameter $k$ after the transient.

Figure 12 and Figure 13. The same considerations as in the previous case can be performed, with the errors even smaller than in the two cases above.

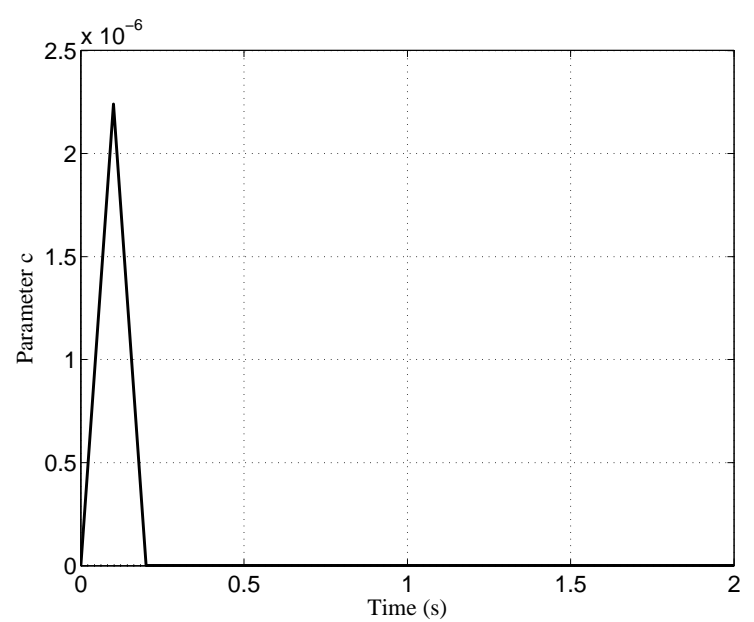

Figure 10: Time evolution of the estimated value $c$.

\section{CONCLUSIONS}

In this paper, the possibility of using a recently constructed micro gripper device for the estimation of the elastic and the damping coefficients of a sample element by pinching it is proved. A classical forgetting factor based recursive least squares algorithms for the parameter estimation is proposed, showing how it is possible to obtain the values of the parameters without the necessity of a specific testing operation, but also during any operative conditions for the gripper. From the methodological point of view, a successive step is the design of a high-performance algorithm for

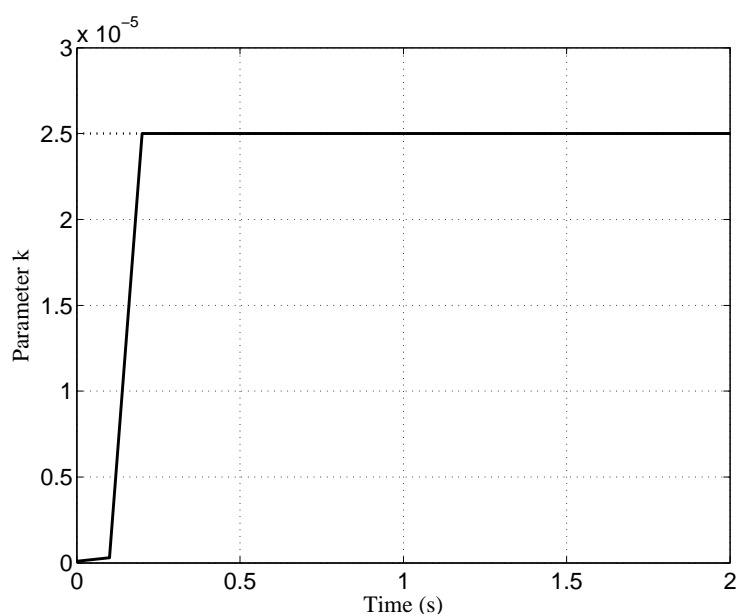

Figure 11: Time evolution of the estimated value $k$.

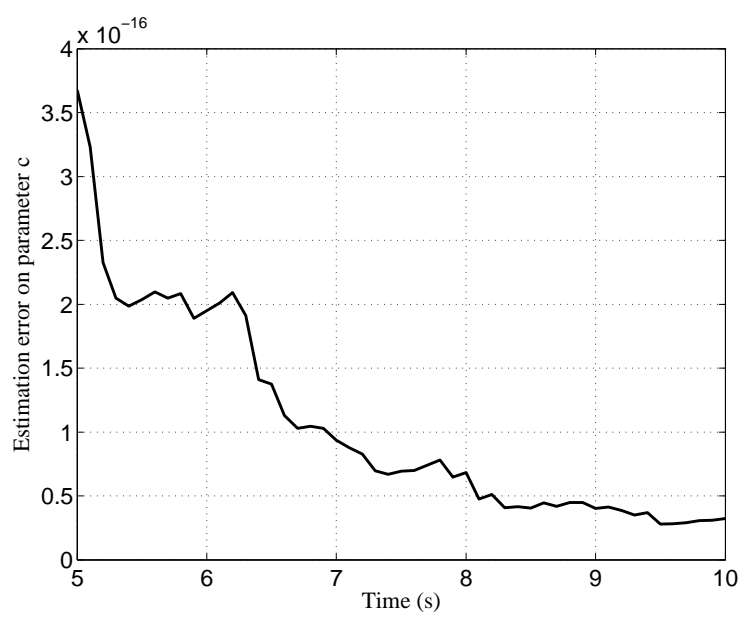

Figure 12: Estimation error on parameter $c$ after the transient.

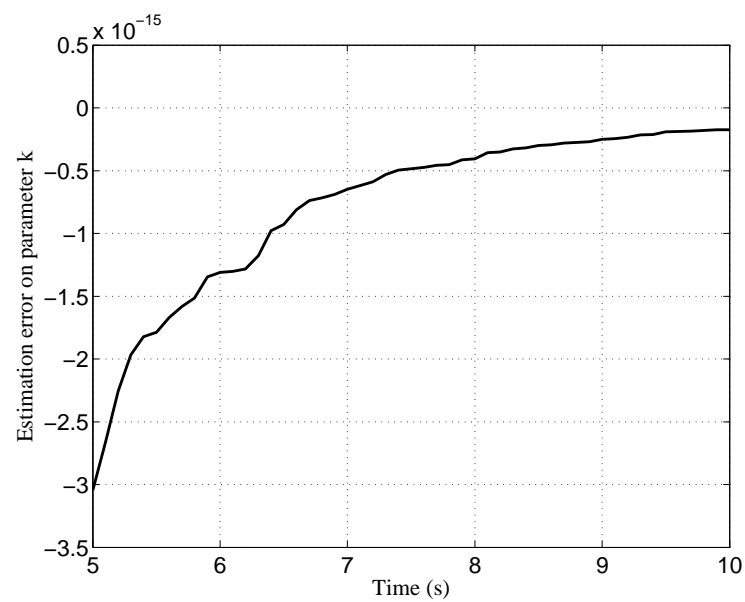

Figure 13: Estimation error on parameter $k$ after the transient. 
the parameters estimation, more robust with respect of the presence of noise and of model parameters uncertainties.

\section{REFERENCES}

Bagolini, A., Bellutti, P., Di Giamberardino, P., Rudas, I.J.and DAndrea, V., Verotti, M., Dochshanov, A., and Belfiore, N. (2018). Stiffness characterization of biological tissues by means of mems-technology based micro grippers under position control. In Mechanisms and Machine Science, volume 49, pages 939 - 947.

Bagolini, A., Ronchin, S., Bellutti, P., Chiste, M., Verotti, M., and Belfiore, N. (2017). Fabrication of novel mems microgrippers by deep reactive ion etching with metal hard mask. IEEE J. Microelectromechanical Syst., 26:926 - 934 .

Boonvisut, P. and Çavuşoǧlu, M. C. (2013). Estimation of soft tissue mechanical parameters from robotic manipulation data. IEEE/ASME Transactions on Mechatronics, 18(5):1602-1611.

Cao, S., Luo, Z., and Quan, C. (2015). Estimation of an objects physical parameter by force sensors of a dualarm robot. In Proceedings of the 2015 IEEE Conference on Robotics and Biomimetics.

Cecchi, R., Verotti, M., Capata, R., Dochshanov, A., Broggiato, G. B., Crescenzi, R., Balucani, M., Natali, S., Razzano, G., Lucchese, F., et al. (2015). Development of micro-grippers for tissue and cell manipulation with direct morphological comparison. Micromachines, 6(11):1710-1728.

Choi, D.-K. (2016). Mechanical characterization of biological tissues: Experimental methods based on mathematical modeling. Biomedical Engineering Letters, 6(3):181-195.

Di Giamberardino, P., Bagolini, A., Bellutti, P., Rudas, I. J., Verotti, M., Botta, F., and Belfiore, N. P. (2018). New mems tweezers for the viscoelastic characterization of soft materials at the microscale. Micromachines, 9(1).

Edsberg, L. E., Cutway, R., Anain, S., and Natiella, J. R. (2000). Microstructural and mechanical characterization of human tissue at and adjacent to pressure ulcers. Journal of Rehabilitation Research and Development, 37(4):463-471.

Flacco, F., De Luca, A., Sardellitti, I., and Tsagarakis, N. G. (2011). Robust estimation of variable stiffness in flexible joints. In in Proc. of 2011 IEEE/RSJ International Conference on Intelligent Robots and Systems.

Fontanelli, G. A., Ficuciello, F., Villani, L., and Siciliano, B. (2017). Modelling and identification of the da vinci research kit robotic arms. In 2017 IEEE/RSJ International Conference on Intelligent Robots and Systems (IROS), pages 1464-1469.

González-Cruz, R. D., Fonseca, V. C., and Darling, E. M. (2012). Cellular mechanical properties reflect the differentiation potential of adipose-derived mesenchymal stem cells. Proceedings of the National
Academy of Sciences of the United States of America, 109(24):E1523-E1529.

Guido, I., Jaeger, M., and Duschl, C. (2011). Dielectrophoretic stretching of cells allows for characterization of their mechanical properties. European Biophysics Journal, 40(3):281-288.

Kiss, M. Z., Varghese, T., and Hall, T. J. (2004). Viscoelastic characterization of in vitro canine tissue. Physics in Medicine and Biology, 49(18):4207-4218.

Lee, S. D. and Jung, S. (2016). A recursive least square approach to a disturbance observer design for balancing control of a single-wheel robot system. 2016 IEEE International Conference on Information and Automation (ICIA), pages 1878-1881.

Ljung, L. (1999). System Identification: Theory for the User. Prentice Hall, 2nd edition.

Lundquist, C. and Schön, T. B. (2009). Recursive identification of cornering stiffness parameters for an enhanced single track model. IFAC Proceedings $\mathrm{Vol}$ umes, 42(10):1726 - 1731. 15th IFAC Symposium on System Identification.

Morrison III, B., Meaney, D. F., and McIntosh, T. K. (1998). Mechanical characterization of an in vitro device designed to quantitatively injure living brain tissue. Annals of Biomedical Engineering, 26(3):381-390.

Nava, A., Mazza, E., Furrer, M., Villiger, P., and Reinhart, W. H. (2008). In vivo mechanical characterization of human liver. Medical Image Analysis, 12(2):203-216.

Nava, A., Mazza, E., Kleinermann, F., Avis, N. J., and McClure, J.and Bajka, M. (2004). Evaluation of the mechanical properties of human liver and kidney through aspiration experiments. Technology and Health Care, 12(3):269-280.

Tavakoli, M., Aziminejad, A., Patel, R., and Moallem, M. (2006). Multi-sensory force/deformation cues for stiffness characterization in soft-tissue palpation. Annual Int. Conference of the IEEE Engineering in Medicine and Biology Society. IEEE Engineering in Medicine and Biology Society, pages 837-840.

Vahidi, A., Stefanopoulou, A., and Peng, H. (2005). Recursive least squares with forgetting for online estimation of vehicle mass and road grade: Theory and experiments. Vehicle System Dynamics, 43(1):31-55.

Verotti, M., Crescenzi, R., Balucani, M., and Belfiore, N. P. (2015). Mems-based conjugate surfaces flexure hinge. Journal of Mechanical Design, 137(1):012301.

Verotti, M., Dochshanov, A., and Belfiore, N. P. (2017). Compliance synthesis of csfh mems-based microgrippers. Journal of Mechanical Design, 139(2):022301.

Wakatsuki, T., Kolodney, M. S., Zahalak, G. I., and Elson, E. L. (2000). Cell mechanics studied by a reconstituted model tissue. Biophysical Journal, 79(5):23532368.

Wilkening, A. and O., I. (2014). Estimation of mass parameters for cooperative human and soft-robots as basis for assistive control of rehabilitation devices. In Proceedings of the RAAD 2014, 23rd International Conference on Robotics in Alpe-Adria-Danube Region. 